\title{
A horta orgânica na escola promovendo saúde e aproximação do aluno com o meio ambiente: um exame bibliográfico
}

\author{
The organic garden at school promoting health and bringing students closer to the \\ environment: a bibliographic examination
}
El huerto orgánico en la escuela que promueve la salud y acerca a los estudiantes al medio ambiente: un examen bibliográfico

Carlos Magno Belonia Moreira ${ }^{1 *}$, Ana Paula Benevenuto dos Santos ${ }^{1}$, Dinalva Silva Cordeiro da Costa $^{1}$, Suellen Aires Ramos${ }^{1}$, Dalva Silva Cordeiro Firmoㄹ, Micheline Scheidegger Fricks Cabellino1, Daniel Franz Reich Magalhães ${ }^{1}$, Marta Alessandra dos Anjos ${ }^{1}$, Telma Maria Paula Rainha Gomes', Cláudia Mara de Oliveira Belonia Vieira1.

\section{RESUMO}

Objetivo: Apontar as relações da horta orgânica no ambiente escolar e a aproximação do aluno com o meio ambiente na promoção da saúde. Revisão Bibliográfica: A escola é o ambiente que aproxima o aluno de muitas áreas do conhecimento, e uma delas é o meio ambiente e a importância dele para a vida humana. Dentro dessa temática extrai-se a questão alimentar, que influi fortemente na saúde do aluno. A horta orgânica na escola é uma das ferramentas de maior engajamento em saúde, já que a alimentação orgânica reduz o risco de doenças futuras, que são causadas por agrotóxicos. Ter uma horta na escola e incluir o aluno na atividade de plantio, colheita e esclarecer os benefícios de se alimentar bem, é além de uma atividade econômica forte, uma atividade de transmissão para a sociedade de como se alimentar bem e o quanto isso pode aumentar a qualidade e a expectativa de vida. Considerações Finais: Compreende-se, que a horta orgânica na escola funciona como instrumento didático para transmitir atividades saudáveis à sociedade além de permitir maneiras mais sustentáveis de produzir alimentos, sem causar prejuízo ao meio ambiente.

Palavras-chave: Serviços de saúde escolar, Meio ambiente e saúde pública, Assistência alimentar.

\begin{abstract}
Objective: To point out the relations of the organic garden in the school environment and the student's approach to the environment in health promotion. Bibliographic Review: The school is the environment that brings students closer to many areas of knowledge, one of which is the environment and its importance for human life. Within this theme, the food issue is extracted, which strongly influences the student's health. The organic garden at school is one of the tools for greater engagement in health, since organic food reduces the risk of future diseases, which are caused by pesticides. Having a vegetable garden at school and including the student in the activity of planting, harvesting and clarifying the benefits of eating well, is in addition to a strong economic activity, an activity for transmitting to society how to eat well and how much it can increase quality and life expectancy. Final Considerations: It is understood that the organic garden at school works as a didactic tool to transmit healthy activities to society, in addition to allowing more sustainable ways of producing food, without causing damage to the environment.
\end{abstract}

Keywords: School health services, Environment and public health, Food assistance.

1 Faculdade Vale do Cricaré (FVC), São Mateus - ES. *E-mail: carlosmagnobelonia@yahoo.com.br 


\section{RESUMEN}

Objetivo: Señalar las relaciones del huerto orgánico en el entorno escolar y el enfoque del alumno hacia el medio ambiente en la promoción de la salud. Revisión Bibliográfica: La escuela es el entorno que acerca a los estudiantes a muchas áreas del conocimiento, una de las cuales es el medio ambiente y su importancia para la vida humana. Dentro de esta temática se extrae el tema de la alimentación, que influye fuertemente en la salud del alumno. El huerto orgánico en la escuela es una de las herramientas para un mayor compromiso con la salud, ya que los alimentos orgánicos reducen el riesgo de futuras enfermedades, que son causadas por pesticidas. Tener un huerto en la escuela e incluir al estudiante en la actividad de plantar, cosechar y aclarar los beneficios de comer bien, se suma a una fuerte actividad económica, una actividad para transmitir a la sociedad cómo comer bien y cuánto puede aumentar calidad y esperanza de vida. Consideraciones Finales: Se entiende que el huerto orgánico en la escuela funciona como una herramienta didáctica para transmitir actividades saludables a la sociedad, además de permitir formas más sostenibles de producir alimentos, sin causar daños al medio ambiente.

Palabras clave: Servicios de salud escolar, Medio ambiente y salud pública, Asistencia alimentaria.

\section{INTRODUÇÃO}

Existem inúmeros motivos que podem ser apresentados na defesa da produção orgânica, mas o principal deles se dá devido às necessidades de proteger a saúde dos produtores e consumidores e proteger o meio ambiente, além do que, a produção de vegetais em sistemas orgânicos está aumentando em todo o mundo. Em particular, os agricultores familiares utilizam esse sistema de produção para se adequar às características da agricultura familiar, e também garantir que os produtos cultivados na mesma área sejam diversificados e que sua dependência de recursos externos seja reduzida (SEDIYAMA MAN, et al., 2014).

O modelo de produção em hortas abre uma infinidade de possibilidades, como a construção alimentar em sociedade e a independência alimentar das famílias. A agricultura familiar, hortas comerciais diversificadas e componentes importantes das hortas caseiras ajudam a aumentar a renda, aumentar a segurança alimentar e melhorar a qualidade dos alimentos. Quando desenvolvidas em nível comunitário, as hortas unem as famílias e constituem um importante meio de socialização e recreação. Eles são usados em escolas da pré-escola ao ensino médio, e são ferramentas para educação acadêmica, educação alimentar e entretenimento ao mesmo tempo (CONRADO TV, et al., 2011).

A forma de integrar esses conhecimentos na educação, requer uma discussão mais aprofundada. A abordagem deve partir do campo teórico ao campo prático, através da interdisciplinaridade. A estratégia interdisciplinar é buscar entender e resolver o problema da união de várias disciplinas, e tentar reconstruir a conexão perdida com a profissão. Nesse caso, diferentes disciplinas podem contribuir para o projeto ou resolver problemas sem se desviar de seus conceitos e métodos. No processo interdisciplinar é imprescindível a cooperação e os intercâmbios entre diferentes campos do conhecimento e campos profissionais, o que pode enriquecer os métodos da disciplina sem afetar uma disciplina ou outra, pois envolve longo prazo e trabalho cooperativo (QUINTAS C, et al., 2016).

Ainda para Quintas C, et al. (2016), sendo as escolas reconhecidas como lugares ideais de aprendizagem, produção e construção de conhecimento, é cada vez mais necessário acompanhar as mudanças contemporâneas, adotar e apoiar as necessidades interdisciplinares atuais de participação na construção de novos conhecimentos. Portanto, à medida que o mundo se torna cada vez mais interligado, interdisciplinar e complexo, ele deve acompanhar as mudanças que compõem os diversos setores da sociedade. A implantação de hortas nos ambientes escolares segue a lógica do acompanhamento das mudanças promovidas nos tempos atuais, já que a alimentação saudável tem se tornado cada dia mais, sinônimo de saúde. 
No campo da nutrição, de acordo com a estrutura do discurso normativo de mudança de hábitos, existe ainda uma estrutura educacional baseada na divulgação de informações científicas, onde a divulgação de informações sobre os prós e os contras dos alimentos e nutrientes reforça essa característica, independentemente da doença determinante Processo e conhecimento popular. Com o objetivo de apoiar as ações de educação alimentar e nutricional nos diferentes setores de forma a integrar estratégias mais amplas de melhoria da qualidade de vida da população e áreas comuns do pensamento e da prática, a política pública do Marco de Referência em Educação Alimentar e Nutricional estabelece que deve ser utilizada na educação alimentar e nutricional métodos e recursos educacionais questionáveis e positivos para facilitar o diálogo com indivíduos e grupos de pessoas, e considerar todas as fases da vida, todas as fases do sistema alimentar, interações e significados (COELHO DEP e BÓGUS CM, 2016).

À primeira vista, as melhorias no ambiente moderno devem levar a uma melhoria do estado nutricional das pessoas e a uma redução do estresse psicológico, melhorando assim a qualidade de vida e a expectativa de vida das pessoas. As razões para os defeitos apontados neste raciocínio simplificado são muitas e só podem resolver esse ângulo de amplo espectro. O ângulo de escolha é o ângulo da nutrição humana, que é diretamente afetado pelo progresso. A transição nutricional populacional trouxe uma nova situação de morbimortalidade em muitas partes do mundo e afetou mais ou menos severamente a saúde humana (FERREIRA SRG, 2010).

Muitos métodos educacionais parecem estar ultrapassados ou não atendendo as demandas sociais da atualidade, devido a isso se propõe a mudança de hábitos comuns e a implantação de novas práticas. $A$ saúde tem se tornado motivo de grande debate social, e como sua promoção pode acontecer no seio da sociedade, gerando mudança de hábitos e consciência social. Devido a isso, é objetivo apontar as relações da horta orgânica no ambiente escolar e a aproximação do aluno com o meio ambiente na promoção da saúde.

\section{REVISÃO BIBLIOGRÁFICA}

A inserção de uma horta em um espaço escolar pode ser uma atividade experimental, interativa e vivenciada em que os alunos podem descobrir objetos fisicamente existentes para que possam obter dados do mundo natural e interagir com ele. Ela pressupõe que os alunos participem de uma determinada situação de ensino, caso em que os dados naturais originais devem ser usados ou exigidos para analisar e pensar. A inserção da horta no espaço escolar é um "laboratório vivo", pois pode realizar diversas atividades pedagógicas e interdisciplinares, integrar os alunos ao ambiente de forma teórica e prática, auxiliar no processo de ensino, proporcionar trabalho coletivo e a sociedade envolvida. A cooperação entre os principais órgãos reflete a importância dos recursos naturais para a sobrevivência dos seres humanos (FRIDRICH GA, 2015).

Justifica-se tal necessidade, devido ao aumento significativo do número de obesos, trabalhar a questão da horta no espaço escolar requer uma análise profunda dos rumos que a sociedade está tomando. Por sua modernidade, facilidade de compra e aumento do poder aquisitivo das famílias, mudou os costumes das pessoas e interferiu diretamente na cultura e nos hábitos. Com isso, as pessoas substituíram os hábitos tradicionais pelos modernos, como por exemplo a compra de alimentos cultivados com agrotóxicos ao invés dos produtos produzidos artesanalmente. Isso tem levado a uma diminuição gradativa do uso de produtos mais saudáveis, o que pode levar à perda do conhecimento tradicional sobre a importância de se alimentar bem como forma de prevenir doenças e aumentar a expectativa de vida. Esses conhecimentos, quando transmitidos no ambiente escolar, promovem uma mudança de olhares e abre portas para que culturas mais saudáveis sejam desenvolvidas no seio social e possam promover o surgimento de novas ideias (ENO EGJ, et al., 2015).

O surgimento de uma nova compreensão da realidade é indispensável, pois promove o renascimento das comunidades da educação, dos negócios, da política, da saúde e da vida cotidiana, de forma que os princípios ambientais e os princípios educacionais sejam incorporados em conjunto. O âmbito da educação, como espaço de construção e socialização do conhecimento, desempenha um papel vital para que os cidadãos se comprometam com a resolução dos problemas do mundo em que vivem (GARUTTI S e PEREIRA RHM, 2017). 
Dentre os programas existentes de promoção à saúde humanitária, está o desenvolvimento dos projetos de implantação de hortas escolares com o objetivo de difundir a prática da horticultura no processo de aplicação de técnicas interdisciplinares, pois ensinam a planejar, implementar e manter a ecologia produtiva do sistema, além de realizar educação alimentar, ensinar o valor nutricional dos vegetais e promover a educação ambiental (GARUTTI S e PEREIRA RHM, 2017).

As atividades realizadas na horta escolar ajudam os alunos a compreender os perigos dos agrotóxicos para a saúde humana e o meio ambiente; entender a necessidade de manter o ambiente escolar; desenvolver o trabalho em equipe e a cooperação; aproxima a conexão com a natureza, porque cada vez menos as crianças nos centros urbanos têm contato com o ambiente natural. Além de reaproveitar materiais como mamadeiras, cartuchos, copos descartáveis etc., também pode-se mudar os hábitos alimentares dos alunos. Essas atividades ajudam a conscientizar as pessoas de que é necessário adotar um estilo de vida com menor impacto ambiental e integrar os alunos aos problemas ambientais encontrados na sociedade (CRIBB SLSP, 2018).

Para os professores, é importante cultivar uma atitude crítica em relação à realidade, às informações e aos valores trazidos pela mídia e de casa como meta entre os alunos. Portanto, o professor precisa entender o assunto e geralmente precisa buscar mais informações para passar aos alunos como parte do meio ambiente, o ser humano faz parte da natureza, portanto, um ambiente saudável é necessário para ter uma vida saudável. Por isso, qualquer dano ao meio ambiente irá prejudicar a saúde de todos. Essa interdependência ilustra a importância de respeitar e proteger a natureza para o bem comum de todos. É fundamental que os professores entendam o que os alunos pensam e sabem sobre seu local de residência, outros lugares e a relação entre eles. Afinal, os alunos têm muitas informações e ideias sobre seu ambiente e o mundo (SCHEFFER LI e SILVA LM, 2016).

A ideia é que todos possam participar da formação e do início das atividades na horta escolar. Toda a comunidade escolar pode colaborar na limpeza do terreno, arrumação dos canteiros e obtenção de sementes indicadas pelo professor responsável pela horta, observando as características do solo, influências climáticas, facilidade de transplante, resistência a "insetos-praga" e, por fim, aspectos que afetam o desenvolvimento das plantas. Este método requer uma perspectiva interdisciplinar, nomeadamente uma compreensão da ecologia, biologia e química. A Interdisciplinaridade utiliza a união de diferentes disciplinas como estratégia para buscar a compreensão e resolução de problemas. Nesse caso, diferentes disciplinas podem contribuir para o projeto ou resolver problemas sem se desviar de seus conceitos e métodos (CRIBB SLSP, 2010).

\section{Os benefícios sociais da horta orgânica na escola}

O ser humano é o responsável pelas rápidas mudanças no meio ambiente, com o passar do tempo, ele desenvolve gradativamente a habilidade de criar objetos e melhorar a qualidade de vida, ferramentas e métodos para minimizar o impacto do desenvolvimento das atividades humanas e usufruir de melhores condições. Como qualquer tipo de ação, o poder humano também traz consequências/desperdícios, sendo necessário, em muitos casos, tratá-los de maneira especial conforme sua origem. O ambiente escolar não difere de outros espaços das cidades ou do campo, e suas atividades resultam também na geração de resíduos sólidos e orgânicos. Além de desempenhar um papel importante na educação, a escola também pode atuar como mediadora na resolução de questões ambientais no cotidiano de trabalho de todas as séries (SILVA ACD, et al., 2015).

$\mathrm{Na}$ literatura científica, alguns estudos avaliaram os benefícios dos alimentos orgânicos para a saúde. Esses estudos afirmam que as dietas orgânicas podem reduzir a exposição das crianças aos pesticidas e ter um impacto positivo na fertilidade, porque muitos pesticidas são desreguladores endócrinos (uma dieta que não contém esses pesticidas pode afetar a fertilidade dos homens). No entanto, como mencionado anteriormente, fazer conexões é difícil porque os estudos populacionais comparam a saúde das pessoas acostumadas a comer alimentos orgânicos com a saúde das pessoas que comem alimentos tradicionais, portanto, há muitas variáveis não controladas (SOUSA AA, et al., 2012). 
O Programa Nacional de Alimentação Escolar (PNAE), administrado pela Fundação Nacional de Desenvolvimento Educacional (FNDE), tem como objetivo melhorar o estado nutricional, promover 0 aprendizado e o desempenho acadêmico dos alunos e formar hábitos alimentares saudáveis. Nessa abordagem, o PNAE parece ser capaz de redefinir as ações estabelecidas pelas escolas, podendo desempenhar um papel estratégico na mudança dos hábitos alimentares dos escolares. Também pode promover a educação em saúde e nutrição e contextualizar a prática da educação nutricional a partir de uma perspectiva mais ampla de construção da consciência cívica (CUNHA E, et al., 2010).

Por meio da grande busca por uma alimentação saudável e da preocupação com a sustentabilidade, o pequeno produtor tem ganhado espaço porque produz seu próprio insumo ecológico, não usa venenos, cultiva produtos saudáveis e o custo de produção não é alto e pode ser vendido a um preço favorável, concentrando a colheita em sua cidade ou região. Portanto, a agricultura em torno do trabalho familiar cria renda para pequenas famílias rurais, combina desenvolvimento ecológico e econômico e incentiva as famílias a continuarem se dedicando à agricultura (SCHIRMANN CB e OSINSKI CRA, 2017).

De acordo com o manual de desenvolvimento municipal elaborado pela Federação Nacional dos Municípios do SEBRAEe (CMN), quando a economia local é fortalecida, oportunidades de trabalho são criadas, a distribuição de renda é melhorada e a renda é aumentada, o desenvolvimento social e econômico do município pode ser ampliado e o bem-estar social promovido através do meio ambiente e do Investimento sustentável, resultando em um amplo ciclo de prosperidade que deve ser iniciado no ambiente escolar, através da disseminação de conhecimentos ambientais e da propagação da sua importância (SCHIRMANN CB e OSINSKI CRA, 2017).

Existem motivos que chamam a atenção para a necessidade de se construir uma sociedade mais consciente acerca da alimentação e como ela é importante para saúde. Alguns desses motivos nascem com as disparidades sociais que surgiram no planeta, aliadas ao crescimento massivo da população, têm atraído grande atenção dos governos para compatibilizar a oferta de alimentos com a demanda de forma sustentável (PAIVA DM, et al., 2019).

A sociedade e o meio ambiente tornaram-se os principais desafios das organizações. Começamos a buscar formas de desenvolvimento sustentável para que 0 atendimento às necessidades atuais nunca permita às gerações futuras passar por necessidades de abastecimento. Devido ao aumento da produção e da produtividade, os problemas econômicos estão relacionados ao reflexo dos efeitos nocivos ao meio ambiente, de modo geral, não consideram os efeitos externos negativos ao meio ambiente, que levam à redução da renda no curto prazo e ao aumento da dependência de fatores externos (PAIVA DM, et al., 2019).

Devido às necessidades humanas é que se faz necessário implementar métodos educativos e instrutivos nas escolas, buscando transmitir esses conhecimentos à sociedade. Os escolares serão responsáveis pelas práticas futuras, eles irão externar os bons ensinamentos que receberem, e a produção orgânica, gerando independência econômica das famílias, sustentabilidade e preservação ambiental, podem ser transmitidas através da prática e a transmissão de conhecimento nas escolas (SILVA ACD, et al., 2015).

É imprescindível abordar que os benefícios vão além dos relacionados à saúde, e todos agem em favor da preservação da vida e da construção de uma sociedade mais justa e humanizada. Um desses benefícios é a redução da desigualdade social, o aumento da arrecadação que por ventura será convertido em programas sociais e benefícios à sociedade (SILVA ACD, et al., 2015).

\section{A horta orgânica na escola promovendo saúde}

A saúde é entendida como um como um estado de completo bem-estar físico, no qual fatores como educação, alimentação, moradia, renda, meio ambiente, trabalho, acesso a bens, serviços e lazer são fatores que determinam seu status. Nesse sentido, levando-se em conta a formação dos alunos, é preciso ampliar o seu estado de saúde, pois isso vai interferir no desenvolvimento intelectual geral do Brasil (CHRISTMANN M e PAVÃO SMO, 2015). 
No ambiente assistencial, um dos níveis de atenção médica são as crianças que sofrem de doenças ou agravos recorrentes de saúde, mas não se caracterizam como doenças crônicas, por isso têm amparo legal nas aulas de internação e adaptação escolar (CHRISTMANN M e PAVÃO SMO, 2015).

O Programa Nacional de Alimentação Escolar tem sido aprimorado de acordo com seus princípios estruturantes para consolidar o direito e o acesso à alimentação adequada e saudável nos espaços escolares, e promover e estimular ações baseadas em pressupostos não alimentares adequados. Por causa de seu conteúdo nutricional, só pode ser entendido e previsto com base em um entendimento estrito da adequação alimentar, e recomendações nutricionais e de energia mínima são fornecidas (BARBOSA NVS, et al., 2013).

Quando essa política foi firmada como parte de uma política pública social e estrutural, atuou-se especificamente na questão alimentar e, ao ser incorporada às suas defesas e princípios, ampliou-se a sustentabilidade. Assim, discutindo a influência dos agrotóxicos pela não utilização da agricultura familiar; na perspectiva da nutrição, saúde e através da alimentação a todos os alunos do ensino fundamental e médio, cuidados promovidos pela prática da alimentação adequada e segura foram se fortalecendo gerando saúde junto da cidadania. Portanto, o plano torna-se uma ação de política pública que visa estabelecer a sustentabilidade, o respeito aos direitos humanos e a cidadania (BARBOSA NVS, et al., 2013).

Nos últimos anos aumentou o interesse pelo consumo de alimentos com maior valor nutricional e menor teor de contaminantes. Além do aumento pela busca de um estilo de vida mais saudável, que também tem promovido o consumo de alimentos orgânicos. O mercado de alimentos orgânicos aumentou em aproximadamente $20 \%$ em taxa de crescimento nos últimos anos (ANDRADE LMS e BERTOLDI MC, 2012).

Conhecer o perfil do consumidor de alimentos orgânicos e os fatores que estimulam ou limitam o seu consumo é fundamental para promover a expansão do mercado para que se adapte às expectativas e necessidades dos consumidores em termos de produtos e serviços (ANDRADE LMS e BERTOLDI MC, 2012).

No campo da nutrição, de acordo com a estrutura do discurso normativo de mudança de hábitos existe ainda uma estrutura educacional baseada na divulgação de informações científicas, em que a divulgação de informações sobre os prós e os contras dos alimentos e nutrientes não leva em consideração o processo de doenças e saúde. Os determinantes do conhecimento popular podem aprimorar esse recurso. Com base nisso, recomenda-se estabelecer uma nova perspectiva para a prática educativa. Embora as hortas nas escolas não sejam novas na educação em saúde ou na educação alimentar e nutricional, ainda há poucas discussões sobre seus métodos e possibilidades educacionais (COELHO DEP e BÓGUS CM, 2016).

A escola é um espaço social para muitas pessoas viverem, trabalharem e estudarem, e promover a saúde dos alunos deve ser uma das metas. A escola é onde os alunos passam a maior parte do tempo, é importante dar atenção especial aos bons hábitos alimentares. Os professores e demais profissionais da escola são exemplos que afetam os alunos e suas famílias (DANELIV L e LEWANDOWSKI H, 2016).

Uma vida saudável deve ser uma prática regular, pois melhorar a qualidade de vida da comunidade escolar também possibilita melhorar o aprendizado. Aprender a fazer as coisas na prática pode permitir que os indivíduos desfrutem de seu próprio plantio, cuidado e compartilhamento no dia seguinte após o jardim, o que parece importante no processo de aprendizagem (DANELIV L e LEWANDOWSKI H, 2016).

Para Accioly E (2009), comer não é apenas um comportamento físico, mas também uma forma de integração social, desta forma, as crianças serão fortemente influenciadas pelas experiências a que obedecem e pelos modelos a seguir no círculo de convivência. A escola desempenha um papel importante na formação de opiniões e no estabelecimento de conceitos, sendo uma referência para a implementação de qualquer plano pessoal de educação e um espaço de convivência e troca de experiências, que pode ser levado ao seio familiar. Portanto, o papel escolar é destaque, por meio do plano de alimentação escolar para estimular bons hábitos alimentares, e buscar escolhas alimentares mais saudáveis e sustentáveis. 


\section{CONSIDERAÇÕES FINAIS}

Uma escola que transmite para a sociedade as boas práticas, que aumenta a qualidade e a expectativa de vida, contribui de forma significativa para a construção de uma sociedade mais equilibrada. A horta na escola é uma forma de disseminar ensinamentos de bem-estar social de maneira prática, colocando o plantio, cultivo e colheita ao alcance do aluno, e incentivando a busca pela produção equiparada. Os benefícios de práticas interdisciplinares nas escolas, vão muito além de unicamente promover saúde, mas também promove inteiração do aluno com o meio ambiente, aumenta a expectativa econômica do produtor e garante a redução da desigualdade social, o que torna o ambiente escolar, próximo do meio ambiente, um caminho para solucionar problemas sociais históricos.

\section{REFERÊNCIAS}

1. ACCIOLY E. A escola como promotora da alimentação saudável. Ciência em tela, 2009; 2(2): 1-9.

2. ANDRADE LMS, BERTOLDI MC. Atitudes e motivações em relação ao consumo de alimentos orgânicos em Belo Horizonte - MG. Braz. J. Food Technol., 2012; 1(1): 31-40.

3. BARBOSA NVS, et al. Alimentação na escola e autonomia - desafios e possibilidades. Ciência \& Saúde Coletiva, 2013; 18(4): 937-945.

4. CHRISTMANN M, PAVÃO SMO. A saúde do escolar cuidada por práticas governamentais: reflexos para a aprendizagem. Rev. educ. PUC-Camp, 2015; 20(3): 265-277.

5. COELHO DEP, BÓGUS CM. Vivências de plantar e comer: a horta escolar como prática educativa, sob a perspectiva dos educadores. Saúde Soc., 2016; 25(3): 761-771.

6. CONRADO TV, et al. Horta Fácil: software para o planejamento, dimensionamento e gerenciamento de hortas em geral. Hortic. bras., 2011; 29(3): 435-440.

7. CRIBB SLSP. Contribuições da educação ambiental e horta escolar na promoção de melhorias ao ensino, à saúde e ao ambiente. Ensino, Saúde e Ambiente, 2010; 3(1): 42-60.

8. CRIBB SLSP. Educação ambiental através da horta escolar: algumas possibilidades. Educação Ambiental em Ação, 2018; 62(1): 1-11.

9. CUNHA E, et al. A alimentação orgânica e as ações educativas na escola: diagnóstico para a educação em saúde e nutrição. Ciência \& Saúde Coletiva, 2010; 15(1): 39-49.

10. DANELIV L, LEWANDOWSKI H. Horta escolar: um instrumento ecoalfabetizador no ensino fundamental. Cadernos PDE, 2016; versão especial: 1-17.

11. ENO EGJ, et al. Horta na escola: incentivo ao cultivo e a interação com o meio ambiente. Revista Eletrônica em Gestão, Educação e Tecnologia Ambiental, 2015; 19(1): 248-253.

12. FERREIRA SRG. Alimentação, nutrição e saúde: avanços e conflitos da modernidade. Ciência e Cultura, 2010; 6294): 31-33.

13. FRIDRICH GA. Horta escolar: como alternativa para educação ambiental. XII Congresso Nacional de Educação, 2015: 35029-35037.

14. GARUTTI S, PEREIRA RHM. Confecção de horta orgânica em um colégio estadual de Maringá -Paraná. Caderno Meio Ambiente e Sustentabilidade, 2017; 10(6): 5-21.

15. PAIVA DM, et al. A agricultura familiar como alternativa sustentável: para um aprimoramento conceitual. Revista Gestão em Foco, 2019; 11(1): 11-24.

16. QUINTAS C, et al. Projeto: Implantação de uma horta em uma escola particular como ferramenta didáticopedagógica. Trabalho (Disciplina de Segurança Alimentar e Nutricional). Universidade de São Paulo, São Paulo, 2016; $17 \mathrm{p}$.

17. SCHEFFER LI, SILVA LM. Horta escolar na busca de uma alimentação saudável. Cadernos PDE, 2016; 1(1): 1-15.

18. SCHIRMANN CB, OSINSKI CRA. Desenvolvimento na agricultura familiar através da produção orgânica: geração de renda aliada a sustentabilidade. VIII Simpósio Iberoamericano em comércio internacional, desenvolvimento e interação regional, 2017: 1-7.

19. SEDIYAMA MAN, et al. Cultivo de hortaliças no sistema orgânico. Revista Ceres, 2014; 61(1): 829-837.

20. SILVA ACD, et al. Horta na escola: sustentabilidade e hábitos saudáveis no município de Cantá-RR. Atas de Saúde Ambiental, 2015; 3(3): 80-89.

21. SOUSA AA, et al. Alimentos orgânicos e saúde humana: estudo sobre as controvérsias. Rev Panam Salud Publica, 2012; 31(6): 513-517. 